\title{
DISTÚRBIO PSÍQUICO MENOR EM MÉDICOS INTENSIVISTAS BRASILEIROS.
}

\author{
$\underline{\text { Aira Benevides Fagundes }}{ }^{1}$; Karoline NerisCedraz²; Carlito Lopes Nascimento \\ Sobrinho $^{3}$ \\ 1. Bolsista FAPESB, Graduando em Medicina, Universidade Estadual de Feira de Santana, e-mail: \\ aira.b.fagundes@gmail.com \\ 2. Bolsista PIBIC/Cnpq, Graduando em Medicina, Universidade Estadual de Feira de Santana, e-mail: \\ karolinecedraz@gmail.com \\ 3. Orientador, Professor Pleno do Departamento de Saúde, Universidade Estadual de Feira de Santana, e-mail: \\ $\underline{\text { mon.ica@terra.com.br }}$
}

PALAVRAS-CHAVE: Prevalência, DPM, Médicos Intensivistas

\section{INTRODUÇÃO}

Entende-se por Unidade de Terapia Intensiva (UTI), uma unidade hospitalar voltada para pacientes que necessitam de cuidados intensivos com a participação de uma equipe especializada e multiprofissional. Diversos estudos apontam que a UTI é o ambiente mais estressante do hospital (AMIB, 2004, NASCIMENTO SOBRINHO, CL, et al., 2010). O trabalho diário do profissional de saúde na UTI exige conhecimento técnico qualificado, habilidades, atenção, raciocínio rápido e controle emocional para lidar com as adversidades que surgem a cada instante, além de atualização científica contínua, frente ao desenvolvimento que a especialidade vem apresentando ao longo dos últimos anos. É preciso ainda ter muita tranqüilidade e preparo psicológico para o apoio aos familiares em momentos de angústia e frustração, que depositam nesses profissionais.

O sofrimento psíquico da equipe hospitalar pode ser identificado pelas jornadas prolongadas e pelo ritmo acelerado de trabalho, a quase inexistência de pausas para descanso ao longo do dia, a intensa responsabilidade por cada tarefa a ser executada, com a pressão de ter "uma vida nas mãos" (BARROS, ET AL., 2008).

Dessa forma, estamos diante, portanto, de uma situação paradoxal, onde a última esperança para a preservação da vida do "ser humano" é depositada em profissionais desgastados, do ponto de vista físico e mental para o exercício profissional, onde o fracasso é irreparável.

Distúrbio Psíquico Menor (DPM) é uma expressão criada por Goldberg \& Huxley (1993) para designar sintomas tais como insônia, fadiga, irritabilidade, esquecimento, dificuldade de concentração e queixas somáticas, que demonstram ruptura do funcionamento normal do indivíduo, mas não configuram categoria nosológica da $10^{\mathrm{a}}$ Classificação Internacional de Doenças (CID-10), bem como dos Manuais de Diagnóstico e Estatística (DSM) da Associação Psiquiátrica Americana (COUTINHO ET AL., 1999). Entretanto, os distúrbios psíquicos menores constituem problema de saúde pública e apresentam impactos econômicos relevantes em função das demandas geradas aos serviços de saúde e do absenteísmo no trabalho (COUTINHO ET AL., 1999).

No Brasil, vários autores têm revelado alta prevalência desses distúrbios em diversos grupos de trabalhadores (ARAÚJO ET AL., 2003; COSTA ET AL., 2002; COSTA E LUDERMIR, 2005; LIMA, 2004; LUDERMIR, 2000) o que pode comprometer as atividades prestadas pelos mesmos, especialmente aquelas relacionadas à saúde, podendo gerar consequiências negativas tanto no plano individual como no coletivo. Dessa forma a identificação precoce de DPM, pode orientar intervenções individuais e coletivas (LIMA, 1999; OMS, 2002). 
Objetivo Geral: estimar a prevalência de Distúrbio Psíquicos Menor em médicos trabalhadores de UTI cadastrados a Associação de Medicina Intensiva Brasileira (AMIB) que participaram do Congresso Brasileiro de Medicina Intensiva (CBMI). Objetivos Específicos: Descrever o perfil sociodemográfico desses trabalhadores. Descrever hábitos de vida relacionados à saúde (uso de bebida alcoólica, tabaco, realização de exames preventivos, prática de atividade física) desses trabalhadores.

\section{MÉTODO}

Foi realizado um estudo epidemiológico de corte transversal, exploratório.

A população estudada foi de Médicos trabalhadores de Unidades de Terapia Intensiva (intensivistas) cadastrados a Associação de Medicina Intensiva Brasileira (AMIB) que participaram do XVIII CBMI realizado no Rio de Janeiro, em 2013 e que consentirem em participar do estudo, após a leitura e assinatura do Termo de Consentimento Livre e Esclarecido (TCLE). Os trabalhadores que participaram do estudo foram identificados por meio dos dados cadastrais existentes na AMIB.

A coleta de dados foi realizada no período de 06 a 09 de novembro, durante o XVIII CBMI, realizado na cidade do Rio de Janeiro, em novembro de 2013, por meio da distribuição de questionário auto-aplicável, acompanhado de Termo de Consentimento Livre e Esclarecido (TCLE).

Os questionários foram acompanhados de carta de apresentação e justificativa do trabalho, referendados pela Associação de Medicina Intensiva Brasileira (AMIB).

Foi utilizado um questionário padronizado, respondido pelos próprios profissionais, não sendo necessário que o mesmo se identificasse. O questionário apresentou de seis blocos de questões: $1^{\circ}$ bloco: identificação geral do entrevistado, destinado a caracterizar os indivíduos integrantes da amostra segundo sexo, idade, especialização, tempo de trabalho profissional, carga horária total trabalhada/semana, turnos de trabalho etc; $2^{\circ}$ bloco: características do ambiente de trabalho percebidas pelos profissionais como nocivas à sua saúde - JobContentQuestionnaire (JCQ); $3^{\circ}$ bloco: referente a qualidade de vida WHOQOLBref; $4^{\circ}$ bloco: queixas de doenças, para avaliar a situação global de saúde dos indivíduos, buscando identificar queixas e sintomas de alguns agravos à saúde, tais como, distúrbios do sono, distúrbios auditivos e visuais, alergias, infecções recorrentes, distúrbios gastrintestinais, dentre outros problemas de saúde mais comuns; $5^{\circ}$ bloco: Avaliação de Distúrbio Psíquico Menor por meio do SRQ-20 (Self ReportQuestionneire) e; $6^{\circ}$ bloco: questões sobre doenças e acidentes de trabalho, problemas de saúde recentes e hábitos de vida e um instrumento de triagem de alcoolismo, (CAGE).

O SRQ-20 foi validado por Mari e Willians em 1986 para a utilização no Brasil, com a finalidade de estudar a morbidade psiquiátrica em instituições de saúde. A versão mais utilizada em estudos de base populacional é composta de 20 questões: 04 sobre sintomas físicos e 16 sobre sintomas psicoemocionais. As respostas são do tipo "sim" ou "não" atribuindo-se, respectivamente, valores de " 1 " e "0". O ponto de corte sugerido por estes autores para DPM é o de 07 respostas positivas. O trabalhador que apresentou escore $\geq 07$ respostas positivas foi considerado positivo e o que apresentou escore $<07$ respostas positivas foi considerado negativo.

Com a devolução dos instrumentos foram construídos dois bancos de dados no EpiData 3.1 para confrontar as informações e identificar possíveis erros de digitação. Após a 
verificação de erros e incongruências foi utilizado para a análise dos dados o programa Statistical Package for the Social Science (SPSS®) 9.0 for Windows, da Sala de Situação e Análise Epidemiológica e Estatística, do Departamento de Saúde, da Universidade Estadual de Feira de Santana (SSAEE/DSAU/UEFS).

\section{RESULTADOS E/OU DISCUSSÃO}

Devido extensão do questionário, houve dificuldade em elaborar o banco de dados no Epidata ocorrendo atraso nos prazos estabelecidos para continuação do projeto. No momento a pesquisa encontra-se em fase de análise de dados com alguns resultados já obtidos.

Nem todos os médicos convidados a participar do estudo responderam o questionário, sendo este fato caracterizado como recusa. Foram estudados 167 médicos intensivistas. Analisando-se a situação sociodemográfica, observou-se que 56,3\% são do sexo masculino. Com relação à idade 48,8\% apresentavam menos de 39 anos. A respeito da situação conjugal classificaram-se os casados e quem possuía união estável como apresentando parceiro e os solteiros e viúvos como sem parceiros, assim observou-se que $67,7 \%$ possuíam parceiro. Quando foram perguntados sobre a presença de filhos 54,5\% informaram ter filhos (Tabela 1).

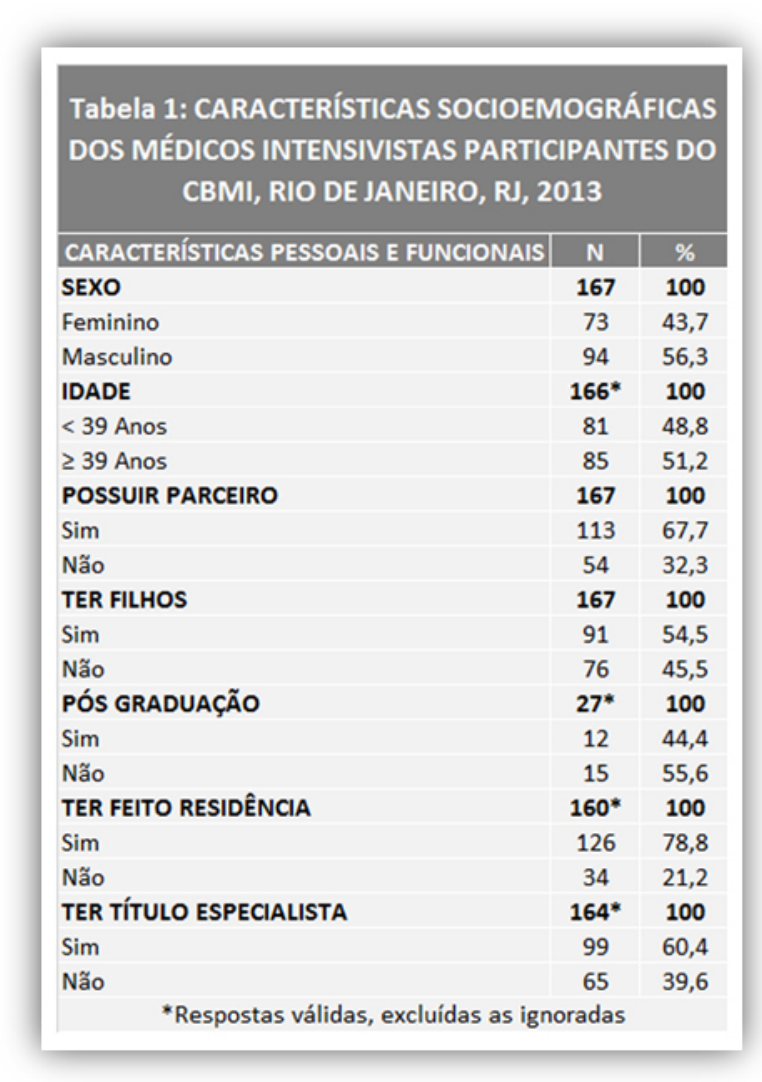

\section{CONSIDERAÇÕES FINAIS}

A pesquisa está em andamento, portanto muito precisa caminhar para obtenção dos resultados almejados.

\section{REFERÊNCIAS BIBLIOGRÁFICAS}


AMIB (Associação de Medicina Intensiva Brasileira). Humanização em cuidados intensivos. São Paulo, Ed. Revinter, 2004.

Araújo, TM et al. Aspectos psicossociais do trabalho e distúrbios psíquicos entre trabalhadoras de enfermagem. Rev Saúde

Pública 2003; 37(4):424-33.

Barros, DS, et al. Médicos plantonistas de unidade de terapia intensiva: perfil sóciodemográfico, condições de trabalho e fatores associados à síndrome de burnout.RevBras Ter Intensiva. 2008; 20(3):235-240.

Costa, JSD et al. Prevalência de distúrbios psiquiátricos menores na cidade de Pelotas, RS. RevBrasEpidemiol 2002; 5(2):164-73.

Costa, AG, Ludermir, AB. TMC e apoio social: estudo em comunidade rural da Zona da Mata de Pernambuco, Brasil. Cad Saúde Pública 2005; 21(1):73-9.

Coutinho, ESF; Almeida-Filho, N; Mari, JJ. Fatores de risco para morbidade psiquiátrica menor: resultado de um estudo transversal em três áreas urbanas do Brasil. RevPsiquiatrClín 1999; 26: 246-56.

Lima, MS; Soares, BGO; Mari, JJ. Saúde e doença mental em Pelotas, RS: um estudo populacional. RevPsiquiatr Calem

1999; 26(5):225-35.

Ludermir, AB; Melo-Filho, DA. Condições de vida e estrutura ocupacional associadas a TMC. Rev Saúde Pública 2002; 36(2):213-21.

Mari, JJ; Willians, P. A validity study of a Psychiatric Screening Questionnaire (SRQ-20) in Primary care in the city of São Paulo. Br J Psychiatry, n. 148, p. 23-26, 1986.

OMS. Relatório sobre a saúde no mundo: nova concepção, nova esperança Lisboa, 2002. 